\title{
Two-dimensional time evolution of beam-plasma instability in the presence of binary collisions
}

\author{
S. F. Tigik ${ }^{1}$, L. F. Ziebell ${ }^{1}$, P. H. Yoon ${ }^{2,3}$, and E. P. Kontar ${ }^{4}$ \\ 1 Instituto de Física, Universidade Federal do Rio Grande do Sul, 91501-970 Porto Alegre, RS, Brazil \\ 2 Institute for Physical Science \& Technology, University of Maryland, College Park, MD 20742, USA \\ 3 School of Space Research, Kyung Hee University, Yongin, 446-701 Gyeonggi, South Korea \\ ${ }^{4}$ School of Physics and Astronomy, University of Glasgow, Glasgow G12 8QQ, UK
}

Received 29 August 2015 / Accepted 21 November 2015

\begin{abstract}
Energetic electrons produced during solar flares are known to be responsible for generating solar type III radio bursts. The radio emission is a byproduct of Langmuir wave generation via beam-plasma interaction and nonlinear wave-wave and wave-particle interaction processes. In addition to type III radio bursts, electrons traveling downwards toward the chromosphere lead to the hard X-ray emission via electron-ion collisions. Recently, the role of Langmuir waves on the X-ray-producing electrons has been identified as important, because Langmuir waves may alter the electron distribution, thereby affecting the X-ray profile. Both Coulomb collisions and wave-particle interactions lead electrons to scattering and energy exchange that necessitates considering the two-dimensional (2D) problem in velocity space. The present paper investigates the influence of binary collisions on the beam-plasma instability development in 2D in order to elucidate the nonlinear dynamics of Langmuir waves and binary collisions. The significance of the present findings in the context of solar physics is discussed.
\end{abstract}

Key words. Sun: radio radiation - Sun: particle emission - methods: numerical

\section{Introduction}

The beam-plasma interaction between the energetic electrons produced during the solar flare and the background coronal plasma is a source of rich nonlinear phenomena. A number of solar radio bursts are associated with non-thermal electron populations and associated Langmuir waves. One of the most important and well-studied phenomena is the generation of solar type-III solar radio bursts (Wild \& McCready 1950; Wild 1950). Fast electrons escaping from active regions in the Sun to the corona and interplanetary space generate Langmuir turbulence, which partially converts to radiation at the local plasma frequency and/or its harmonic(s). A nonlinear conversion process involves sophisticated wave decay and wave scattering. This is the well-known plasma emission, and it is the basic radio emission mechanism for type III radio bursts and for the reverse slope bursts (see, e.g., Tang \& Moore 1982; Dennis et al. 1984).

The first theory of plasma emission was put forth by Ginzburg \& Zhelezniakov (1958) and many modifications and improvements have been made over the past six decades (Tsytovich 1967; Kaplan \& Tsytovich 1968; Zheleznyakov \& Zaitsev 1970; Melrose 1982; Goldman \& Dubois 1982; Goldman 1983; Cairns 1987; Robinson \& Cairns 1998; Mel'Nik et al. 1999; Kontar 2001; Ledenev et al. 2004; Li et al. 2005, 2006a,b, 2008a,b; Li \& Cairns 2013; Ratcliffe \& Kontar 2014). Although the essential theoretical framework based upon EM weak turbulence theory, which describes the entire process starting from the beam-generated Langmuir turbulence to the radiation generation, was available, complete numerical solution of the entire set of EM weak turbulence equations have not been done until quite recently, when Ziebell et al. (2014a,b,c, 2015) numerically solved the complete equations of EM weak turbulence theory for the first time. It should be mentioned that a few authors carried out direct EM particle-in-cell (PIC) simulations to characterize the nonlinear behavior of the plasma emission process (Kasaba et al. 2001; Karlický \& Vandas 2007; Rhee et al. 2009a,b; Umeda 2010; Ganse et al. 2012a,b, 2014). The full numerical solution of the analytical EM weak turbulence equations by Ziebell et al. (2014a, 2015) complements these PIC simulation efforts.

The type III radio bursts are not the only process of importance associated with solar flares. For electrons traveling down to the chromosphere, they generate X-rays via bremsstrahlung when they collide with plasma. The approximate treatment of the electron dynamics by only considering the Coulomb collisions is known as the thick target model (e.g., Holman et al. 2011, as a recent review). Under such a simplifying assumption, Brown (1971) and Syrovatskii \& Shmeleva (1972) analyzed the dynamics of electron distribution and the related bremsstrahlung X-ray spectrum from accelerated/injected electrons. In the literature, however, there are discussions of the importance of Langmuir wave generation on the underlying non-thermal electron energy distribution. Emslie \& Smith (1984) first considered the effects of wave generation on the electron beam propagation toward the chromosphere. Hamilton \& Petrosian (1987) and McClements (1987, 1989) reconsidered the same problem but found that the influence of wave-particle interactions was insignificant for 
stationary electron injection. However, recent works by Hannah et al. (2009, 2013) and Karlický \& Kontar (2012) demonstrate that the collective effects are sufficiently significant after all, especially for transient sources of nonthermal electrons, such that without these effects, the solar hard X-ray spectra measured by RHESSI spacecraft may not be interpreted correctly. Zharkova \& Siversky (2011) also studied a similar problem. Moreover, Kontar et al. (2012) have shown that the presence of plasma inhomogeneity and/or nonlinear wave-wave interactions can lead to an overestimated number and energy of energetic electrons accelerated in the corona even in the case of stationary electron injection.

As noted above, the problem of flare-generated nonthermal electrons and X-ray emission from the same source that also generates type III radio bursts is a complicated one, which requires simultaneous time-dependent treatment of various processes that include Coulomb collisions, collective Langmuir wave generation, and nonlinear wave-wave interactions. In addition, the evolution of electrons not only in energy but also in collisional pitch-angle scattering is important for interpreting some X-ray observations (e.g., MacKinnon 1991; Jeffrey et al. 2014).

The purpose of the present paper is not to revisit the plasma emission, which has recently been discussed by Ziebell et al. (2014a, 2015) and by Ratcliffe \& Kontar (2014) in application to flaring loops, or to address the issue of the influence of collective processes on the X-ray generation by bremsstrahlung per se, which was addressed by Hannah et al. (2009) and Kontar et al. (2012). Instead, we address a fundamental 2D plasma physics problem that may be related to both the type-III-generating electrons and the X-ray-generating non-thermal electrons. That is, we consider the influence of both Coulomb collisions and the collective effects in two-dimensional (2D) electron beam-plasma interaction problem, taking only the evolution of the particle distribution and of the spectra of electrostatic waves into account, without incorporating the effects associated to electromagnetic oscillations.

Unlike previous works related to this type of analysis where the effects of Coulomb collisions have normally been ignored (Ziebell et al. 2008), the present paper includes electron-electron and electron-ion collisions. On the other hand, unlike the previous works related to the solar X-ray problem where, in addition to collisional effects, quasilinear effects are included considering one-dimensional (1D) wave excitation, we now consider the nonlinear dynamics associated with the wave scattering and decay in two dimensions, along with the collisional dynamics. The 2D evolution is particularly important since collisional scattering and collisional energy loss operate on the same time scales. Moreover, radioemission and Langmuir wave evolution depend on angular distribution of plasma waves. The objective with such relatively limited analysis that only includes electrostatic waves is to gather information about the effect of collisions on the time evolution of the beam-plasma system, on the relative roles of collisions and nonlinear mechanisms of three-wave decay and wave-particle scattering, and on the possibly different time scales of the different physical processes when evolving self-consistently. The information to be obtained with such study may be useful for future analysis of the time evolution of more complex processes, in which other nonlinear mechanisms have to be taken into account, such as the actual production of fundamental and harmonic emission that characterize type III emissions and the complicated interaction between energetic electrons, background plasma, and waves, which is associated to the solar X-ray emission.
The structure of the paper is as follows. In Sect. 2 we briefly describe the theoretical formulation and the setup for the numerical analysis. In Sect. 3 we present and discuss the results of the numerical analysis. Finally, in Sect. 4 we conclude the paper and comment on the results obtained.

\section{Theoretical formulation and numerical setup}

The wave kinetic equations for $L$ and $S$ waves that describe quasilinear process as well as nonlinear decay and scattering processes are given in terms of the spectral wave energy density, $I_{\boldsymbol{k}}^{\sigma L}=\left\langle E_{L}^{\sigma 2}(\boldsymbol{k})\right\rangle$ and $I_{\boldsymbol{k}}^{\sigma S}=\left\langle E_{S}^{\sigma 2}(\boldsymbol{k})\right\rangle$, where $E_{L}^{\sigma}(\boldsymbol{k})$ and $E_{S}^{\sigma}(\boldsymbol{k})$ represent the spectral electric field component associated with $L$ and $S$ waves, respectively, and where $\sigma= \pm 1$ stands for the sign of wave phase velocity. The wave kinetic equations for these waves are given by Yoon (2006) and Ziebell et al. (2008):

$$
\begin{aligned}
\frac{\partial I_{\boldsymbol{k}}^{\sigma L}}{\partial t} & =\frac{\pi \omega_{\mathrm{p}}^{2}}{k^{2}} \int \mathrm{d} \boldsymbol{v} \delta\left(\sigma \omega_{\boldsymbol{k}}^{L}-\boldsymbol{k} \cdot \boldsymbol{v}\right)\left(\frac{n_{0} e^{2}}{\pi} F_{\mathrm{e}}(\boldsymbol{v})\right. \\
& \left.+\sigma \omega_{\boldsymbol{k}}^{L} I_{\boldsymbol{k}}^{\sigma L} \boldsymbol{k} \cdot \frac{\partial F_{\mathrm{e}}(\boldsymbol{v})}{\partial \boldsymbol{v}}\right)+\frac{\pi e^{2}}{2 T_{\mathrm{e}}^{2}} \sum_{\sigma^{\prime}, \sigma^{\prime \prime}= \pm 1} \sigma \omega_{\boldsymbol{k}}^{L} \\
& \times \int \mathrm{d} \boldsymbol{k}^{\prime} \frac{\mu_{\boldsymbol{k}-\boldsymbol{k}^{\prime}}\left(\boldsymbol{k} \cdot \boldsymbol{k}^{\prime}\right)^{2}}{k^{2} k^{\prime 2}\left|\boldsymbol{k}-\boldsymbol{k}^{\prime}\right|^{2}} \delta\left(\sigma \omega_{\boldsymbol{k}}^{L}-\sigma^{\prime} \omega_{\boldsymbol{k}^{\prime}}^{L}-\sigma^{\prime \prime} \omega_{\boldsymbol{k}-\boldsymbol{k}^{\prime}}^{S}\right) \\
& \times\left[\sigma \omega_{\boldsymbol{k}}^{L} I_{\boldsymbol{k}^{\prime}}^{\sigma^{\prime} L} \frac{\left.I_{\boldsymbol{k}-\boldsymbol{k}^{\prime}}^{\sigma^{\prime \prime} S}-\left(\sigma^{\prime} \omega_{\boldsymbol{k}^{\prime}}^{L} \frac{I_{\boldsymbol{k}-\boldsymbol{k}^{\prime}}^{\sigma^{\prime \prime} S}}{\mu_{\boldsymbol{k}-\boldsymbol{k}^{\prime}}}+\sigma^{\prime \prime} \omega_{\boldsymbol{k}-\boldsymbol{k}^{\prime}}^{L} I_{\boldsymbol{k}^{\prime}}^{\sigma^{\prime} L}\right) I_{\boldsymbol{k}}^{\sigma L}\right]}{}\right. \\
& -\frac{\pi e^{2}}{m_{\mathrm{e}}^{2} \omega_{\mathrm{p}}^{2}} \sum_{\sigma^{\prime}= \pm 1} \int \mathrm{d} \boldsymbol{k}^{\prime} \int \mathrm{d} \boldsymbol{v} \frac{\left(\boldsymbol{k} \cdot \boldsymbol{k}^{\prime}\right)^{2}}{k^{2} k^{\prime 2}} \delta\left[\sigma \omega_{\boldsymbol{k}}^{L}-\sigma^{\prime} \omega_{\boldsymbol{k}^{\prime}}^{L}\right. \\
& \left.-\left(\boldsymbol{k}-\boldsymbol{k}^{\prime}\right) \cdot \boldsymbol{v}\right] \times\left(\frac{\hat{n} e^{2}}{\pi \omega_{\mathrm{pe}}^{2}} \sigma \omega_{\boldsymbol{k}}^{L}\left(\sigma^{\prime} \omega_{\boldsymbol{k}^{\prime}}^{L} I_{\boldsymbol{k}}^{\sigma L}-\sigma \omega_{\boldsymbol{k}}^{L} I_{\boldsymbol{k}^{\prime}}^{\sigma^{\prime} L}\right) F_{i}\right. \\
& \left.-\frac{m_{\mathrm{e}}}{m_{i}} \sigma \omega_{\boldsymbol{k}}^{L} I_{\boldsymbol{k}^{\prime}}^{\sigma^{\prime} L} I_{\boldsymbol{k}}^{\sigma L}\left(\boldsymbol{k}-\boldsymbol{k}^{\prime}\right) \cdot \frac{\partial F_{i}}{\partial \boldsymbol{v}}\right),
\end{aligned}
$$$$
\frac{\partial}{\partial t} \frac{I_{\boldsymbol{k}}^{\sigma S}}{\mu_{\boldsymbol{k}}}=\frac{\pi \mu_{\boldsymbol{k}} \omega_{\mathrm{p}}^{2}}{k^{2}} \int \mathrm{d} \boldsymbol{v} \delta\left(\sigma \omega_{\boldsymbol{k}}^{S}-\boldsymbol{k} \cdot \boldsymbol{v}\right)\left[\frac{n_{0} e^{2}}{\pi}\left(F_{\mathrm{e}}+F_{i}\right)\right.
$$$$
\left.+\sigma \omega_{\boldsymbol{k}}^{L} \frac{I_{\boldsymbol{k}}^{\sigma S}}{\mu_{\boldsymbol{k}}} \boldsymbol{k} \cdot \frac{\partial}{\partial \boldsymbol{v}}\left(F_{\mathrm{e}}+\frac{m_{\mathrm{e}}}{m_{i}} F_{i}\right)\right]+\frac{\pi e^{2}}{4 T_{\mathrm{e}}^{2}} \sum_{\sigma^{\prime}, \sigma^{\prime \prime}= \pm 1} \sigma \omega_{\boldsymbol{k}}^{L}
$$$$
\times \int \mathrm{d} \boldsymbol{k}^{\prime} \frac{\mu_{\boldsymbol{k}}\left[\boldsymbol{k}^{\prime} \cdot\left(\boldsymbol{k}-\boldsymbol{k}^{\prime}\right)\right]^{2}}{k^{2} k^{\prime 2}\left|\boldsymbol{k}-\boldsymbol{k}^{\prime}\right|^{2}} \delta\left(\sigma \omega_{\boldsymbol{k}}^{S}-\sigma^{\prime} \omega_{\boldsymbol{k}^{\prime}}^{L}-\sigma^{\prime \prime} \omega_{\boldsymbol{k}-\boldsymbol{k}^{\prime}}^{L}\right)
$$$$
\times\left(\sigma \omega_{\boldsymbol{k}}^{L} I_{\boldsymbol{k}^{\prime}}^{\sigma^{\prime} L} I_{\boldsymbol{k}-\boldsymbol{k}^{\prime}}^{\sigma^{\prime \prime} L}-\left(\sigma^{\prime} \omega_{\boldsymbol{k}^{\prime}}^{L} I_{\boldsymbol{k}-\boldsymbol{k}^{\prime}}^{\sigma^{\prime \prime} L}+\sigma^{\prime \prime} \omega_{\boldsymbol{k}-\boldsymbol{k}^{\prime}}^{L} I_{\boldsymbol{k}^{\prime}}^{\sigma^{\prime} L}\right) \frac{I_{\boldsymbol{k}}^{\sigma S}}{\mu_{\boldsymbol{k}}}\right),
$$

where $\omega_{\mathrm{p}}=\left(4 \pi n_{0} e^{2} / m_{\mathrm{e}}\right)^{1 / 2}$ is the electron plasma frequency, and e, $m_{\mathrm{e}}$, and $n_{0}$ stand for the unit electric charge, electron mass, and the ambient particle number density, respectively. The dispersion relations for $L$ and $S$ modes are well-known:

$$
\begin{aligned}
& \omega_{\boldsymbol{k}}^{L}=\omega_{\mathrm{p}}\left(1+\frac{3}{2} k^{2} \lambda_{\mathrm{D}}^{2}\right), \\
& \omega_{\boldsymbol{k}}^{S}=\omega_{\mathrm{p}} \frac{k \lambda_{\mathrm{D}}}{\left(1+k^{2} \lambda_{\mathrm{D}}^{2}\right)^{1 / 2}}\left(\frac{m_{\mathrm{e}}}{m_{i}}\right)^{1 / 2}\left(1+\frac{3 T_{i}}{T_{\mathrm{e}}}\right)^{1 / 2},
\end{aligned}
$$

where $v_{\mathrm{e}}=\left(2 T_{\mathrm{e}} / m_{\mathrm{e}}\right)^{1 / 2}$ is the electron thermal speed, and $\lambda_{\mathrm{D}}=v_{\mathrm{e}} /\left(\sqrt{2} \omega_{\mathrm{p}}\right)$ is the electron Debye length, with $T_{\mathrm{e}}$ being 
the electron temperature. In (1) and (2) we have also introduced a quantity

$\mu_{k}=|k|^{3} \lambda_{\mathrm{D}}^{3}\left(\frac{m_{\mathrm{e}}}{m_{i}}\right)^{1 / 2}\left(1+\frac{3 T_{i}}{T_{\mathrm{e}}}\right)^{1 / 2}$.

The first terms on the righthand sides of (1) and (2) describe the spontaneous emission and quasilinear effects. The second terms contain the energy conservation condition, $\delta\left(\sigma \omega_{\boldsymbol{k}}^{L}-\sigma^{\prime} \omega_{\boldsymbol{k}^{\prime}}^{L}-\right.$ $\left.\sigma^{\prime \prime} \omega_{k-k^{\prime}}^{S}\right)$, for $L$ mode and a similar three-wave resonance condition for $S$ mode, and describe the three-wave decay processes. The third term in (1) contains the nonlinear wave-particle resonance condition $\delta\left[\sigma \omega_{\boldsymbol{k}}^{L}-\sigma^{\prime} \omega_{\boldsymbol{k}^{\prime}}^{L}-\left(\boldsymbol{k}-\boldsymbol{k}^{\prime}\right) \cdot \boldsymbol{v}\right]$, and it depicts the scattering of $L$ waves by the ions. We have neglected the scattering effects for (2), which rules the evolution of $S$ waves, since the scattering processes involving $S$ waves are extremely slow processes.

For the time evolution of the particle distribution, we consider the effects of spontaneous fluctuation, the quasilinear diffusion effect, and the effect of binary collisions. The particle kinetic equation is thus given by

$$
\begin{aligned}
& \frac{\partial F_{a}(\boldsymbol{v})}{\partial t}=\frac{\partial}{\partial v_{i}}\left(A_{i}(\boldsymbol{v}) F_{a}(\boldsymbol{v})+D_{i j}(\boldsymbol{v}) \frac{\partial F_{a}(\boldsymbol{v})}{\partial v_{j}}\right)+\sum_{b} \theta_{a b}\left(F_{a}, F_{b}\right), \\
& A_{i}(\boldsymbol{v})=\frac{e_{a}^{2}}{4 \pi m_{a}} \int \mathrm{d} \boldsymbol{k} \frac{k_{i}}{k^{2}} \sum_{\sigma= \pm 1} \sigma \omega_{\boldsymbol{k}}^{L} \delta\left(\sigma \omega_{\boldsymbol{k}}^{\alpha}-\boldsymbol{k} \cdot \boldsymbol{v}\right), \\
& D_{i j}(\boldsymbol{v})=\frac{\pi e_{a}^{2}}{m_{a}^{2}} \int \mathrm{d} \boldsymbol{k} \frac{k_{i} k_{j}}{k^{2}} \sum_{\sigma= \pm 1} \delta\left(\sigma \omega_{\boldsymbol{k}}^{\alpha}-\boldsymbol{k} \cdot \boldsymbol{v}\right) I_{\boldsymbol{k}}^{\sigma \alpha}, \\
& \theta_{a b}\left(F_{a}, F_{b}\right)=-\frac{2 \pi n_{0 a} n_{0 b} e_{a}^{2} e_{b}^{2} \ln \Lambda}{m_{a}} \frac{\partial}{\partial v_{i}} \\
& \quad \times \int \mathrm{d} \boldsymbol{v}^{\prime} U_{i j}\left(\frac{F_{a}(\boldsymbol{v})}{m_{b}} \frac{\partial F_{b}\left(\boldsymbol{v}^{\prime}\right)}{\partial v_{j}^{\prime}}-\frac{F_{b}\left(\boldsymbol{v}^{\prime}\right)}{m_{a}} \frac{\partial F_{a}(\boldsymbol{v})}{\partial v_{j}}\right) \\
& U_{i j}=\frac{w^{2} \delta_{i j}-w_{i} w_{j}}{w^{3}}, \quad \boldsymbol{w}=\boldsymbol{v}-\boldsymbol{v}^{\prime} .
\end{aligned}
$$

The term with coefficient $A_{i}$ describes the effects of spontaneous fluctuations, and the term with coefficient $D_{i j}$ rules the quasilinear diffusion process. The symbol $\alpha$ represents either $L$ or $S$, depending on whether the particle species $a$ in the above particle kinetic equations stands for $a=e$ (electrons) or $a=i$ (ions). The quantity $\theta_{a b}\left(F_{a}, F_{b}\right)$ represents the binary collisions of particles of species $a$ with particles of species $b$. The quantity $\ln \Lambda$ is the Coulomb logarithm.

In the set of wave kinetic Eqs. (1) and (2), we do not (yet) include a collisional damping term. The collisional effect is only incorporated through the particle kinetic equation (3), which contains the collision operator $\sum_{b} \theta_{a b}\left(F_{a}, F_{b}\right)$. Upon linearization of the governing particle kinetic equation and coupling to the wave equation, then the collision frequency $v_{\text {coll }}$ broadens (Dupree 1966; Ishihara \& Hirose 1985; Bian et al. 2014; Pécseli 2014) the wave-particle resonance conditions, $\omega-\boldsymbol{k} \cdot \boldsymbol{v}$, where $v_{\text {coll }}$ is the effective collision frequency that is obtained from the particle equation. In this way, the collisional damping rate for the collective wave phenomena is calculated by the particle collision term in an indirect way. However, a more complete treatment should contain explicit collisional damping term for the wave kinetic equation. In a recently submitted work, Yoon et al. (2015) address this issue by reformulating the standard weak turbulence theory to include contributions from those electrostatic fluctuation spectra $(\omega, k)$ for which $\omega$ and $k$ do not satisfy the plasma wave dispersion relations. They show that these so-called "noneigenmode" contributions, which are typically ignored in the literature, are not only responsible for the same collision integral $\sum_{b} \theta_{a b}\left(F_{a}, F_{b}\right)$ in the particle kinetic equation, as in Eq. (3), but also they lead to the collisional damping term, as well as the electrostatic analog of the bremsstrahlung. One could, however, include the collisional damping term heuristically, as in the recent paper by Ratcliffe \& Kontar (2014).

We introduce the following dimensionless variables and definitions:

$$
\begin{aligned}
& z \equiv \frac{\omega}{\omega_{\mathrm{pe}}}, \quad \tau \equiv \omega_{\mathrm{pe}} t, \quad \boldsymbol{q} \equiv \frac{\boldsymbol{k} v_{t \mathrm{e}}}{\omega_{\mathrm{pe}}}, \quad \boldsymbol{u} \equiv \frac{\boldsymbol{v}}{v_{t \mathrm{e}}} \\
& \mu_{\boldsymbol{q}}^{L}=1, \quad \mu_{\boldsymbol{q}}^{T}=1, \quad \mu_{\boldsymbol{q}}^{S}=\frac{q^{3}}{2^{3 / 2}} \sqrt{\frac{m_{\mathrm{e}}}{m_{i}}}\left(1+\frac{3 T_{i}}{T_{\mathrm{e}}}\right)^{1 / 2}, \\
& \lambda_{\mathrm{De}}^{2}=\frac{T_{\mathrm{e}}}{4 \pi \hat{n} e^{2}}=\frac{v_{t e}^{2}}{2 \omega_{\mathrm{pe}}^{2}}, \quad g=\frac{1}{2^{3 / 2}(4 \pi)^{2} \hat{n} \lambda_{\mathrm{De}}^{3}}, \\
& F_{a}(\boldsymbol{u})=v_{t \mathrm{e}}^{3} f_{a}(\boldsymbol{u}), \quad \mathcal{E}_{\boldsymbol{q}}^{\sigma \alpha}=\frac{(2 \pi)^{2} g}{m_{\mathrm{e}} v_{t \mathrm{e}}^{2}} \frac{I_{\boldsymbol{k}}^{\sigma \alpha}}{\mu_{\boldsymbol{k}}^{\alpha}}
\end{aligned}
$$

In terms of the normalized variables and quantities, the equation for Langmuir $(L)$ waves is expressed as

$$
\begin{aligned}
\frac{\partial \mathcal{E}_{\boldsymbol{q}}^{\sigma L}}{\partial \tau}= & \left\{\mu_{\boldsymbol{q}}^{L} \frac{\pi}{q^{2}} \int \mathrm{d} \boldsymbol{u} \delta\left(\sigma z_{\boldsymbol{q}}^{L}-\boldsymbol{q} \cdot \boldsymbol{u}\right)\right. \\
& \left.\times\left(g F_{\mathrm{e}}(\boldsymbol{u})+\left(\sigma z_{\boldsymbol{q}}^{L}\right) \boldsymbol{q} \cdot \frac{\partial F_{\mathrm{e}}(\boldsymbol{u})}{\partial \boldsymbol{u}} \mathcal{E}_{\boldsymbol{q}}^{\sigma L}\right)\right\}_{L q l} \\
& +\left\{2 \sigma \mu _ { \boldsymbol { q } } ^ { L } z _ { \boldsymbol { q } } ^ { L } \sum _ { \sigma ^ { \prime } , \sigma ^ { \prime \prime } } \int \mathrm { d } \boldsymbol { q } ^ { \prime } \frac { \mu _ { \boldsymbol { q } ^ { \prime } } ^ { L } \mu _ { \boldsymbol { q } - \boldsymbol { q } ^ { \prime } } ^ { S } ( \boldsymbol { q } \cdot \boldsymbol { q } ^ { \prime } ) ^ { 2 } } { q ^ { 2 } q ^ { \prime 2 } | \boldsymbol { q } - \boldsymbol { q } ^ { \prime } | ^ { 2 } } \left(\sigma z_{\boldsymbol{q}}^{L} \mathcal{E}_{\boldsymbol{q}^{\prime}}^{\sigma^{\prime} L} \mathcal{E}_{\boldsymbol{q}^{\sigma_{-} \boldsymbol{q}^{\prime}}}^{\sigma^{\prime \prime} S}\right.\right. \\
& \left.-\sigma^{\prime} z_{\boldsymbol{q}^{\prime}}^{L} \mathcal{E}_{\boldsymbol{q}-\boldsymbol{q}^{\prime}}^{\sigma^{\prime \prime} S} \mathcal{E}_{\boldsymbol{q}}^{\sigma L}-\sigma^{\prime \prime} z_{\boldsymbol{q}-\boldsymbol{q}^{\prime}}^{L} \mathcal{E}_{\boldsymbol{q}^{\prime}}^{\sigma^{\prime} L} \mathcal{E}_{\boldsymbol{q}}^{\sigma L}\right) \\
& \left.\times \delta\left(\sigma z_{\boldsymbol{q}}^{L}-\sigma^{\prime} z_{\boldsymbol{q}^{\prime}}^{L}-\sigma^{\prime \prime} z_{\boldsymbol{q}-\boldsymbol{q}^{\prime}}^{S}\right)\right\}_{L d L S}+\left\{\sigma z_{\boldsymbol{q}}^{L} \sum_{\sigma^{\prime}} \int \mathrm{d} \boldsymbol{q}^{\prime}\right. \\
& \times \int \mathrm{d} \boldsymbol{u} \frac{\mu_{\boldsymbol{q}}^{L} \mu_{\boldsymbol{q}^{\prime}}^{L}\left(\boldsymbol{q} \cdot \boldsymbol{q}^{\prime}\right)^{2}}{q^{2} q^{\prime 2}} \delta\left[\sigma z_{\boldsymbol{q}}^{L}-\sigma^{\prime} z_{\boldsymbol{q}^{\prime}}^{L}-\left(\boldsymbol{q}-\boldsymbol{q}^{\prime}\right) \cdot \boldsymbol{u}\right](4) \\
& \times\left[g\left(\sigma z_{\boldsymbol{q}}^{L} \mathcal{E}_{\boldsymbol{q}^{\prime}}^{\sigma^{\prime} L}-\sigma^{\prime} z_{\boldsymbol{q}^{\prime}}^{L} \mathcal{E}_{\boldsymbol{k}}^{\sigma L}\right)\left[F_{\mathrm{e}}(\boldsymbol{u})+F_{i}(\boldsymbol{u})\right]\right. \\
& \left.\left.+\frac{m_{\mathrm{e}}}{m_{i}} \mathcal{E}_{\boldsymbol{q}^{\prime}}^{\sigma^{\prime} L} \mathcal{E}_{\boldsymbol{q}}^{\sigma L}\left(\boldsymbol{q}-\boldsymbol{q}^{\prime}\right) \cdot \frac{\partial F_{i}(\boldsymbol{u})}{\partial \boldsymbol{u}}\right]\right\}_{L s L L}
\end{aligned}
$$

The equation for ion-acoustic $(S)$ waves is likewise expressed in terms of dimensionless quantities,

$$
\begin{aligned}
\frac{\partial \mathcal{E}_{\boldsymbol{q}}^{\sigma S}}{\partial \tau}= & \left\{\mu _ { \boldsymbol { q } } ^ { S } \frac { \pi } { q ^ { 2 } } \int \mathrm { d } \boldsymbol { u } \delta ( \sigma z _ { \boldsymbol { q } } ^ { S } - \boldsymbol { q } \cdot \boldsymbol { u } ) \left[g\left[F_{\mathrm{e}}(\boldsymbol{u})+F_{i}(\boldsymbol{u})\right]\right.\right. \\
& \left.\left.+\left(\sigma z_{\boldsymbol{q}}^{L}\right)\left(\boldsymbol{q} \cdot \frac{\partial F_{\mathrm{e}}(\boldsymbol{u})}{\partial \boldsymbol{u}}+\frac{m_{\mathrm{e}}}{m_{i}} \boldsymbol{q} \cdot \frac{\partial F_{i}(\boldsymbol{u})}{\partial \boldsymbol{u}}\right) \mathcal{E}_{\boldsymbol{q}}^{\sigma S}\right]\right\}_{S q l} \\
& +\left\{\sigma z_{\boldsymbol{q}}^{L} \sum_{\sigma^{\prime}, \sigma^{\prime \prime}} \int \mathrm{d} \boldsymbol{q}^{\prime} \frac{\mu_{\boldsymbol{q}}^{S} \mu_{\boldsymbol{q}^{\prime}}^{L} \mu_{\boldsymbol{q}-\boldsymbol{q}^{\prime}}^{L}\left[\boldsymbol{q}^{\prime} \cdot\left(\boldsymbol{q}-\boldsymbol{q}^{\prime}\right)\right]^{2}}{q^{2} q^{\prime 2}\left|\boldsymbol{q}-\boldsymbol{q}^{\prime}\right|^{2}}\right. \\
& \times\left(\sigma z_{\boldsymbol{q}}^{L} \mathcal{E}_{\boldsymbol{q}^{\prime}}^{\sigma^{\prime} L} \mathcal{E}_{\boldsymbol{q}-\boldsymbol{q}^{\prime}}^{\sigma^{\prime \prime} L}-\sigma^{\prime} z_{\boldsymbol{q}^{\prime}}^{L} \mathcal{E}_{\boldsymbol{q}-\boldsymbol{q}^{\prime}}^{\sigma^{\prime \prime} L} \mathcal{E}_{\boldsymbol{q}}^{\sigma S}-\sigma^{\prime \prime} z_{\boldsymbol{q}-\boldsymbol{q}^{\prime}}^{L} \mathcal{E}_{\boldsymbol{q}^{\prime}}^{\sigma^{\prime} L} \mathcal{E}_{\boldsymbol{q}}^{\sigma S}\right) \\
& \left.\times \delta\left(\sigma z_{\boldsymbol{q}}^{S}-\sigma^{\prime} z_{\boldsymbol{q}^{\prime}}^{L}-\sigma^{\prime \prime} z_{\boldsymbol{q}_{-\boldsymbol{q}^{\prime}}}^{L}\right)\right\}_{S \mathrm{~d} L L}
\end{aligned}
$$


The dimensionless particle kinetic equation is given by

$$
\begin{aligned}
\frac{\partial F_{a}(\boldsymbol{u})}{\partial \tau}= & \frac{e_{a}^{2}}{e^{2}} \frac{m_{\mathrm{e}}^{2}}{m_{a}^{2}} \sum_{\sigma} \sum_{\alpha=L, S} \int \mathrm{d} \boldsymbol{q}\left(\frac{\boldsymbol{q}}{q} \cdot \frac{\partial}{\partial \boldsymbol{u}}\right) \mu_{\boldsymbol{q}}^{\alpha} \delta\left(\sigma z_{\boldsymbol{q}}^{\alpha}-\boldsymbol{q} \cdot \boldsymbol{u}\right) \\
& \times\left(g \frac{m_{a}}{m_{\mathrm{e}}} \frac{\sigma z_{\boldsymbol{q}}^{L}}{q} F_{a}(\boldsymbol{v})+\mathcal{E}_{\boldsymbol{q}}^{\sigma \alpha} \frac{\boldsymbol{q}}{q} \cdot \frac{\partial F_{a}(\boldsymbol{u})}{\partial \boldsymbol{u}}\right) \\
& +\sum_{b} \theta_{a b}\left(F_{a}, F_{b}\right)
\end{aligned}
$$

Finally, the dispersion relations in dimensionless form are given by

$$
\begin{aligned}
& z_{\boldsymbol{q}}^{L}=\left(1+\frac{3}{2} q^{2}\right)^{1 / 2}, \\
& z_{\boldsymbol{q}}^{S}=\frac{q A}{\left(1+q^{2} / 2\right)^{1 / 2}}, \quad A=\frac{1}{\sqrt{2}}\left(\frac{m_{\mathrm{e}}}{m_{i}}\right)^{1 / 2}\left(1+3 \frac{T_{i}}{T_{\mathrm{e}}}\right)^{1 / 2},
\end{aligned}
$$

where $v_{t \mathrm{e}}=\left(2 T_{\mathrm{e}} / m_{\mathrm{e}}\right)^{1 / 2}$ is the electron thermal speed, and $\lambda_{\mathrm{De}}$ is the electron Debye length, with $T_{\mathrm{e}}$ being the electron temperature.

For further reference, in the equation for the $L$ waves, Eq. (5), the term that describes the spontaneous emission and the quasilinear effects has been denoted $L q l$, the term that describes the three-wave decay involving $L$ and $S$ waves has been denoted $L \mathrm{~d} L S$, and the term describing scattering involving $L$ waves has been denoted $L s L L$. In the equation for the evolution of $S$ waves, namely Eq. (6), the first term describes the spontaneous emission and quasilinear effects and is denoted $S q l$, and the second term describes the three-wave decay involving $L$ waves and is denoted $S \mathrm{~d} L L$.

In the equation for the particle distribution functions (7), the term with $g$ describes the effects of spontaneous fluctuations, and the term with the velocity derivative describes the quasilinear diffusion process.

For a detailed derivation of the above equations without the term that represents the collisional effects, the reader is referred to Yoon $(2000,2005,2006)$. The collisional term is essentially the Landau collision integral, which is well known in the literature (e.g., Lifshitz \& Pitaevskii 1981; Karney 1986; Jeffrey et al. 2014, see also Appendix A). Assuming that both ion and electron background distributions are Maxwellian distributions, the kinetic equation describing the evolution of electron distribution function $F_{a}$ in fully ionized hydrogen plasma can be written in spherical coordinates $(v, \theta)$,

$$
\begin{aligned}
\sum_{b} \theta_{a b}\left(F_{a}, F_{b}\right)= & \frac{\Gamma}{2 v^{2}}\left\{\frac{\partial}{\partial v}\left(2 v G(u) \frac{\partial f}{\partial v}+4 u^{2} G(u) f\right)\right. \\
& \left.+\frac{1}{v} \frac{\partial}{\partial \mu}\left(\left(1-\mu^{2}\right)[\operatorname{erf}(u)-G(u)] \frac{\partial f}{\partial \mu}\right)\right\} \\
& +\frac{\Gamma}{2 v^{3}} \frac{\partial}{\partial \mu}\left(\left(1-\mu^{2}\right) \frac{\partial f}{\partial \mu}\right),
\end{aligned}
$$

where, $\mu=v_{\|} / v=\cos (\theta), u=\sqrt{m v^{2} / 2 k_{\mathrm{B}} T_{\mathrm{e}}}, \Gamma=4 \pi n e^{4} \ln \Lambda / m_{e}^{2}$, $\operatorname{erf}(u)$ is the error function, and $G(u)$ is the Chandrasekhar function, given by

$G(u)=\frac{\operatorname{erf}(u)-u \operatorname{erf}^{\prime}(u)}{2 u^{2}}$.
The first term on the righthand side of Eq. (7) describes electronelectron collisions, while the second is due to electron scattering by ions. One can combine the collisional terms (Eq. (7)) with the collective terms (Eq. (6)) and define generalized friction and diffusion coefficients.

The initial electron distribution function is assumed to be made of a Maxwellian background and a forward beam distribution with number density $n_{\mathrm{f}}$. In $2 \mathrm{D}$, the assumed distribution function is given as

$$
\begin{aligned}
F_{\mathrm{e}}(\boldsymbol{v}, 0)= & \frac{1-n_{\mathrm{f}} / n_{0}}{\pi v_{t \mathrm{e}}^{2}} \exp \left(-\frac{v_{\perp}^{2}}{v_{t \mathrm{e}}^{2}}-\frac{\left(v_{\|}-v_{0}\right)^{2}}{v_{t \mathrm{e}}^{2}}\right) \\
& +\frac{n_{\mathrm{f}} / n_{0}}{\pi v_{t f}^{2}} \exp \left(-\frac{v_{\perp}^{2}}{v_{t f}^{2}}-\frac{\left(v_{\|}-v_{f}\right)^{2}}{v_{t f}^{2}}\right) .
\end{aligned}
$$

Here $v_{t \mathrm{e}}^{2}=2 T_{\mathrm{e}} / m_{\mathrm{e}}$ and $v_{t f}^{2}=2 T_{f} / m_{\mathrm{e}}$ are the background and the beam thermal velocities squared, respectively. The quantities $v_{0}$ and $v_{f}$ are the drift velocities of the background and forward beam, respectively. The background drift velocity $v_{0}$ is such that it guarantees zero net drift velocity for the electron distribution, i.e., $v_{0}=-\left(v_{f} n_{\mathrm{f}}\right) /\left(n_{0}-n_{\mathrm{f}}\right)$. The initial ion distribution in $2 \mathrm{D}$ is given by $F_{\mathrm{i}}(\boldsymbol{v})=\left(m_{\mathrm{i}} / 2 \pi T_{\mathrm{i}}\right) \exp \left[-m_{\mathrm{i}} v^{2} /\left(2 T_{\mathrm{i}}\right)\right]$, where $T_{\mathrm{i}}$ and $m_{\mathrm{i}}$ are the ion temperature and the proton mass, respectively. For the present study, we assume that the ion distribution remains constant along the time evolution.

The intensities of $L$ and $S$ waves are initialized by balancing the spontaneous and induced emissions, taking the background population into account:

$$
\begin{aligned}
I_{\boldsymbol{k}}^{\sigma L}(0)= & \frac{T_{\mathrm{e}}}{4 \pi^{2}} \frac{1}{1+3 k^{2} \lambda_{\mathrm{D}}^{2}} \\
I_{\boldsymbol{k}}^{\sigma S}(0)= & \frac{T_{\mathrm{e}}}{4 \pi^{2}} k^{2} \lambda_{\mathrm{D}}^{2} \sqrt{\frac{1+k^{2} \lambda_{\mathrm{D}}^{2}}{1+3 k^{2} \lambda_{\mathrm{D}}^{2}}} \\
& \times \frac{\int \mathrm{d} \boldsymbol{v} \delta\left(\sigma \omega_{\boldsymbol{k}}^{S}-\boldsymbol{k} \cdot \boldsymbol{v}\right)\left(F_{\mathrm{e}}+F_{\mathrm{i}}\right)}{\int \mathrm{d} \boldsymbol{v} \delta\left(\sigma \omega_{\boldsymbol{k}}^{S}-\boldsymbol{k} \cdot \boldsymbol{v}\right)\left[F_{\mathrm{e}}+\left(T_{\mathrm{e}} / T_{\mathrm{i}}\right) F_{\mathrm{i}}\right]}
\end{aligned}
$$

\section{Numerical analysis}

The set of Eqs. (4), (5), and (8)-(10) have been solved in 2D wave number space and $2 \mathrm{D}$ velocity space by employing a splitting method with fixed time step for the equation for evolution of the electron distribution and a fourth-order Runge-Kutta method for the wave equations. The $2 \mathrm{D}$ version for the particle equation is shown by Eq. (A.11), with the coefficients coming from weak turbulence theory as given by (A.12), and the collision term given by Eqs. (A.9) and (A.10). Similar equations for the wave evolution can be obtained by converting Eqs. (4) and (5) to 2D coordinates. For all the cases, we have used $\Delta \tau=0.1$. We employed a $51 \times 51$ grid for $k_{\perp}$ and $k_{\|}$ with $0<k_{\perp} v_{t \mathrm{e}} / \omega_{\mathrm{p}}<0.6$, and $0<k_{\|} v_{t \mathrm{e}} / \omega_{\mathrm{p}}<0.6$. For the velocities, we used a $51 \times 101$ grid for $v_{\perp} / v_{t e}$ and $v_{\|} / v_{t e}$, covering the velocity range $0<v_{\perp} / v_{t e}<12$ and $-12<v_{\|} / v_{t e}<12$. For the subsequent numerical solutions to be discussed, we assume a forward beam with the same temperature as the background population, $T_{f} / T_{\mathrm{e}}=1.0$, electron-to-ion temperature ratio $T_{\mathrm{e}} / T_{\mathrm{i}}=7.0$, plasma parameter $\left(n_{0} \lambda_{\mathrm{D}}^{3}\right)^{-1}=5.0 \times 10^{-3}$, and 
electron temperature, such that $v_{t \mathrm{e}}^{2} / c^{2}=4.0 \times 10^{-3}$. We also assume $v_{f} / v_{t \mathrm{e}}=5.0$ and $n_{\mathrm{f}} / n_{0}=1.0 \times 10^{-3}$, a value that is relatively high for the case of solar wind beam electrons, but has been assumed in order to reduce the computational time required to obtain the numerical results.

The characteristics of the grids used for the numerical analyses were also chosen as a compromise between the resolution obtained and the performance of the numerical code. The grid used in wave number space resolves some fundamental details in the wave spectra, such as (a) the primary peak that grows due to quasilinear interaction between $\mathrm{L}$ waves and the beam particles in the region of the positive derivative of the distribution function and (b) the peak corresponding to backwardly propagating $\mathrm{L}$ waves, which are generated by nonlinear interaction between $\mathrm{L}$ and $S$ waves. In velocity space, the grid used allows for a smooth description of the distribution function and of the beam, including the important region of transition between the core distribution and the beam. Even with use of the chosen grids, the code needs to run for a few days in a personal computer with updated technology. Owing to the 2D nature of the problem investigated, a twofold increase in resolution would require nearly a fourfold increase in computational time. We have reached the conclusion that such a costly enhanced resolution would not be needed to describe the relevant features of the time evolution of the system under investigation.

As one application of the numerical code, we studied the time evolution of the system in two situations. The first situation neglects the influence of collisions, which can be easily done just by turning off the collisional term in the equation for the evolution of the electron distribution function. In the second situation, we took the effect of binary collisions into account. For comparison, we also added some results that show the time evolution of the distribution function that is only subject to collisions.

Figure 1 shows the time evolution of electron velocity distribution function by plotting $F_{\mathrm{e}}$ in $2 \mathrm{D}$ velocity space at different time steps corresponding to $\omega_{\text {pe }} t=500,1000,2000$, $5000,10^{4}, 1.5 \times 10^{4}$, and $2 \times 10^{4}$. The lefthand panels depict solutions with only collisions; the middle panels correspond to the self-consistent solution without collisional effects, as in the type III beam case (Ziebell et al. 2008); and the righthand panels show solutions that contain both collisional effects and self-consistent Langmuir wave dynamics. As the lefthand panels show, the collisional relaxation of the beam leads to the systematic energy losses and isotropization of the beam. Eventually, the beam merges with the Maxwellian background. In contrast, collisionless quasilinear relaxation, appearing in the middle panels, shows that slowing down the beam proceeds via the velocity space plateau formation.

The total electron distribution undergoes some heating, but the overall shape of the distribution is quite different from the collision-only case. In these middle panels, it is noticeable that an X-shaped band of irregular features appears near the core of the distribution in the late stages of the time evolution. These irregular features in the distribution function are apparently connected with irregular features that appear near the edge of the grid representing the spectrum of $\mathrm{L}$ waves and must be due to accumulation of numerical imprecision. These irregular features do not spread and do not produce any perceptible effect on the relevant features of the distribution, such as the formation of the plateau joining the core and the beam distributions or the overall broadening of the velocity distribution.

In accordance with this interpretation, one sees that the irregular features do not appear in the left- and righthand panels, since they are washed out by the effect of collisions. At the end of the computational period, it is seen that the net electron distribution is still anisotropic, with the 2D distribution elongated along the beam direction. In the case of collisions plus the Langmuir wave dynamics (the righthand panels), the early evolution is similar to the purely quasilinear case, but the later evolution is affected by the Coulomb collisional dynamics. Such a combined beamplasma dynamical interaction showing the transition from the collective dynamics to collisional dynamics has not been done in the literature.

In the context of the solar applications, the third panels are similar to those considered (e.g., Hamilton \& Petrosian 1987; McClements 1989; Hannah et al. 2009; Hannah \& Kontar 2011; Zharkova \& Siversky 2011), in that both the Coulomb collisional dynamics and self-consistent Langmuir wave dynamics are considered. The above references, however, also heuristically included collisional damping term for the Langmuir waves, whereas in the present approach, the collisional effects influence the Langmuir dynamics indirectly and only through the electron distribution function evolution. In spite of this caveat, however, our work is more general in the sense that we consider twodimensional dynamics and also allow nonlinear wave processes, such as the decay interaction and nonlinear wave-particle scattering similar to Kontar et al. (2012) and Karlický \& Kontar (2012). Also, the above references employed simplified Coulomb collisional integral for the particles, whereas our numerical solution is based upon the Landau operator. In the context of solar X-ray, however, inhomogeneity effects is important. In addition, for the solar X-ray problem different initial electron velocity distributions should be adopted, such as the power-law distribution. We do not have inhomogeneity effects (see, e.g., Kontar \& Pécseli 2002), and our choice of initial electron distribution is a simple Gaussian core plus a drifting beam. As such, our calculation is not meant to represent the solar X-ray problem. Instead, our interest has been on understanding the 2D evolution.

Figure 2 shows the spectrum of Langmuir (L) waves, as a function of normalized components of a wave vector in 2D space for several values of the normalized time between $\tau=500$ and $\tau=20000$. Since the purely collisional dynamics involves no waves by default, the Langmuir spectrum is absent in that case. As such, Fig. 2 only shows two situations. The lefthand panels show Langmuir wave spectra when only self-consistent collective effects are considered without the collisional terms in the particle equation, and the righthand panels solutions that contain both collisional dynamics and self-consistent wave dynamics in the particle kinetic equations. Of course, for both cases, the ionsound wave intensity is also self-consistently calculated, but the results are not shown. Both cases exhibit similar early-time dynamics of the Langmuir wave time evolution.

The forward-propagating "primary" Langmuir waves are the direct result of bump-on-tail instability by the electron beam. For $\omega_{\mathrm{pe}} t=500$, the nonlinear decay and scattering off thermal ions (these are nonlinear processes depicted in the wave kinetic equations) already begin to produce weak backward-traveling Langmuir waves. These "backscattered" Langmuir waves form a semi-arc shaped spectra as time proceeds, eventually distributing the wave momentum in the quasi circular area in 2D wave number space. For later dynamics, such as beyond $\omega_{\text {pe }}=15000$, however, the evolution of the wave spectra for two cases are quite distinct. In the pure collective dynamic case, one notices that there is an appreciable gap along the parallel wave number; in contrast, in the general case where both collective dynamics and collisions are included, the primary Langmuir waves undergo spreading at a wave vector azimuthal angle so that the 

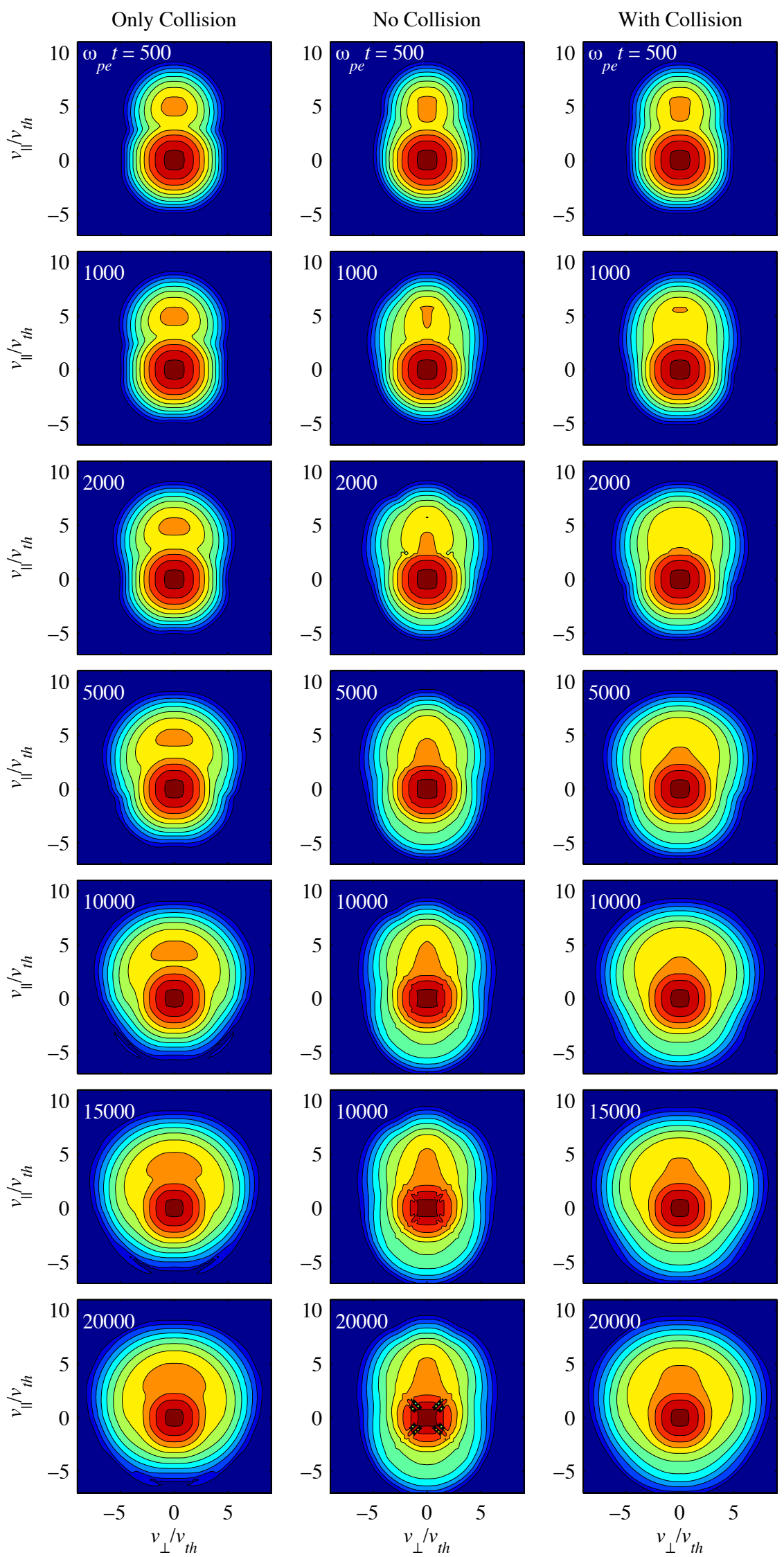

Fig. 1. Time evolution of electron velocity distribution function is shown in 2D velocity space plot at different time steps. The lefthand panels depict solutions with only collisions (as in the thick target approximation), the middle panels correspond to the self-consistent solution without collisional effects (as in the type III beam case), and the right panels show solutions that contain both collisional dynamics and self-consistent Langmuir wave dynamics. 
S. F. Tigik et al.: 2D evolution of beam-plasma instability
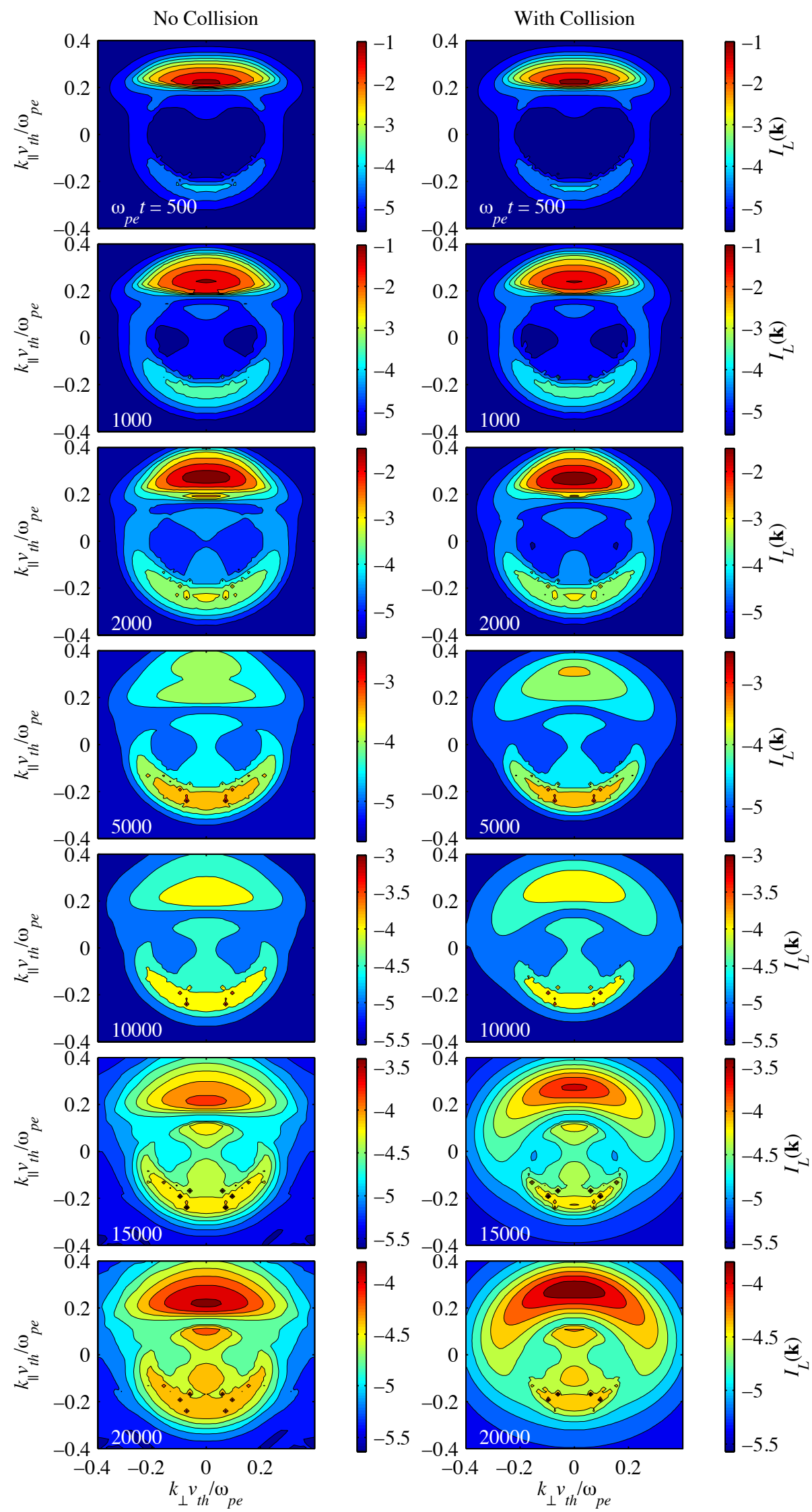

Fig. 2. Time evolution of Langmuir wave spectrum as a 2D wave number space plot at different time steps. The lefthand panels depict solutions with only self-consistent collective effects without the collisional terms in the particle equation, and the right ones show solutions that contain both collisional dynamics and self-consistent wave dynamics. 
wave spectrum appears to be much more symmetric throughout the semi-circular area.

In the context of solar applications, Hamilton \& Petrosian (1987), McClements (1989), Hannah et al. (2009), Hannah \& Kontar (2011), and Zharkova \& Siversky (2011), solved selfconsistent Langmuir wave kinetic equations. Kontar et al. (2012) also demonstrate numerically that nonlinear wave-wave interactions are important. However, since these authors did not consider nonlinear mode coupling physics in $2 \mathrm{D}$, the Langmuir spectrum in such a scheme does not give the wave vector pitch angle evolution. The evolution in pitch angle affects the level of Langmuir waves and generally reduces peak values of spectral energy density of plasma waves.

\section{Final remarks}

The energetic electrons produced during the solar flare lead to the generation of solar type III solar radio bursts and/or reverse slope bursts when the fast electrons escape the acceleration region. Electrons propagating downwards are responsible for reverse slope bursts (e.g., Tang \& Moore 1982) that generate Langmuir waves in collisional plasma. These electrons traveling down towards the chromosphere are responsible for X-rays emitted via bremsstrahlung (see, e.g., Holman et al. 2011). There have been recent discussions (Hannah et al. 2009; Hannah \& Kontar 2011; Zharkova \& Siversky 2011; Kontar et al. 2012) that show that the collective Langmuir-wave dynamics of the $\mathrm{X}$-ray generating electrons may be important for interpretations of X-ray spectra from RHESSI spacecraft (Lin et al. 2002).

In the present paper, we have addressed a fundamental plasma physics problem that is related to both the type III-generating electrons and the X-ray-generating nonthermal electrons. We considered the influence of Coulomb collisions and the self-consistent collective effects in the electron beam-plasma interaction problem in $2 \mathrm{D}$ velocity space. In the past, the present authors investigated the electron-Langmuir wave collective dynamics, which is relevant to the type III burst electrons, by ignoring the effects of Coulomb collisions (Ziebell et al. 2008). On the other hand, early works on solar X-ray emission ignored the collective dynamics and only considered collisional dynamics. Later works include wave-particle interactions and included Langmuir wave generation, but they only considered the 1D problem, although they included other effects relevant to the solar X-ray problem, such as the inhomogeneity and phenomenological collisional damping. In contrast, we considered the full nonlinear dynamics in the wave kinetic equation without explicit collisional damping (yet), but which included nonlinear wave-particle scattering and three-wave decay interactions in $2 \mathrm{D}$ wave number space.

Upon comparing the electron distribution evolution among three situations, namely, one in which only the collisional effects are included, another with only collective dynamics, and the third in which both collisions and waves are incorporated, we found that the early dynamics in the general case follows the collective dynamical pattern, whereas for later times, the dynamics closely matches the collisional case. This transitional behavior has not been demonstrated clearly in the literature (Fig. 1). The comparison between results obtained considering the collisionless weak turbulent dynamics and the collisional weak turbulent dynamics shows that for $\tau \simeq 2000$, a normalized time for which the primary Langmuir peak excited by the beam is fully developed and the backward propagating Langmuir peak is already well established, the effect of collisions is already noticeable, leading to a wider plateau in the distribution function and increased tendency to isotropization, as compared to the collisionless case. For a much longer evolution time, the results show that the distribution function obtained in the collisional case is clearly more isotropic than was obtained in the case without the effect of collisions and also show a noticeable decrease in the energetic tail. Considering the effect of the wave spectra as well, the general comment is that collisions lead to a wider angular spread of both Langmuir waves and electron beam on comparable time scales.

When we compared the wave dynamics between the pure collective case and the general case (for collision only case there is no wave), we found that the late time evolution of the wave spectra also shows a marked difference between the two cases. Specifically, the general case in which both collisions and collective effects were considered shows more symmetric Langmuir wave spectrum at late time periods. The overall conclusion to be drawn from both analyses of the time evolution of the velocity distribution function and of the spectrum of Langmuir waves is that the collisional processes, even if irrelevant on the time scale of the development of quasilinear processes, may lead to effects that are comparable in magnitude to effects of the nonlinear processes on a time scale that is compatible to the time scale of evolution of nonlinear processes in space plasmas.

As noted, the actual application of the present analysis to the solar X-ray problem requires additional effects that are not included here: inhomogeneous density profile, explicit collisional damping terms in the wave kinetic equations, and probably different initial non-thermal electron distribution functions. However, we believe that future applications can be built upon the present formalism.

Acknowledgements. This work was partially supported by the Brazilian agencies CNPq and FAPERGS. P.H.Y. acknowledges support by the BK21 plus program through the National Research Foundation (NRF) funded by the Ministry of Education of Korea, and NSF Grant Nos. AGS1138720 and AGS1242331. E.P.K. was supported by an STFC consolidated grant.

\section{References}

Bian, N. H., Kontar, E. P., \& Ratcliffe, H. 2014, J. Geophys. Res. (Space Phys.), 119,4239

Brown, J. C. 1971, Sol. Phys., 18, 489

Cairns, I. H. 1987, J. Plasma Phys., 38, 169

Dennis, B. R., Benz, A. O., Ranieri, M., \& Simnett, G. M. 1984, Sol. Phys., 90, 383

Dupree, T. H. 1966, Phys. Fluids, 9, 1773

Emslie, A. G., \& Smith, D. F. 1984, ApJ, 279, 882

Gaffey, Jr., J. D. 1976, J. Plasma Phys., 16, 149

Ganse, U., Kilian, P., Spanier, F., \& Vainio, R. 2012a, ApJ, 751, 145

Ganse, U., Kilian, P., Vainio, R., \& Spanier, F. 2012b, Sol. Phys., 280, 551

Ganse, U., Kilian, P., Spanier, F., \& Vainio, R. 2014, A\&A, 564, A15

Ginzburg, V. L., \& Zhelezniakov, V. V. 1958, Sov. Astron., 2, 653

Goldman, M. V. 1983, Sol. Phys., 89, 403

Goldman, M. V., \& Dubois, D. F. 1982, Phys. Fluids, 25, 1062

Hamilton, R. J., \& Petrosian, V. 1987, ApJ, 321, 721

Hannah, I. G., \& Kontar, E. P. 2011, A\&A, 529, A109

Hannah, I. G., Kontar, E. P., \& Sirenko, O. K. 2009, ApJ, 707, L45

Hannah, I. G., Kontar, E. P., \& Reid, H. A. S. 2013, A\&A, 550, A51

Holman, G. D., Aschwanden, M. J., Aurass, H., et al. 2011, Space Sci. Rev., 159, 107

Ishihara, O., \& Hirose, A. 1985, Phys. Fluids, 28, 2159

Jeffrey, N. L. S., Kontar, E. P., Bian, N. H., \& Emslie, A. G. 2014, ApJ, 787, 86

Kaplan, S. A., \& Tsytovich, V. N. 1968, Sov. Astron., 11, 956

Karlický, M., \& Kontar, E. P. 2012, A\&A, 544, A148

Karlický, M., \& Vandas, M. 2007, Planet. Space Sci., 55, 2336

Karney, C. F. F. 1986, Comp. Phys. Rep., 4, 183

Kasaba, Y., Matsumoto, H., \& Omura, Y. 2001, J. Geophys. Res., 106, 18693 
S. F. Tigik et al.: 2D evolution of beam-plasma instability

Kontar, E. P. 2001, Sol. Phys., 202, 131

Kontar, E. P., \& Pécseli, H. L. 2002, Phys. Rev. E, 65, 066408

Kontar, E. P., Ratcliffe, H., \& Bian, N. H. 2012, A\&A, 539, A43

Ledenev, V. G., Zverev, E. A., \& Starygin, A. P. 2004, Sol. Phys., 222, 299

Li, B., \& Cairns, I. H. 2013, J. Geophys. Res. (Space Phys.), 118, 4748

Li, B., Willes, A. J., Robinson, P. A., \& Cairns, I. H. 2005, Phys. Plasmas, 12, 012103.1

Li, B., Robinson, P. A., \& Cairns, I. H. 2006a, Phys. Plasmas, 13, 092902

Li, B., Robinson, P. A., \& Cairns, I. H. 2006b, Phys. Rev. Lett., 96, 145005

Li, B., Cairns, I. H., \& Robinson, P. A. 2008a, J. Geophys. Res. (Space Phys.), 113,6104

Li, B., Cairns, I. H., \& Robinson, P. A. 2008b, J. Geophys. Res. (Space Phys.), 113,6105

Lifshitz, E. M., \& Pitaevskii, L. P. 1981, Physical kinetics (Oxford: Pergamon Press)

Lin, R. P., Dennis, B. R., Hurford, G. J., et al. 2002, Sol. Phys., 210, 3

MacKinnon, A. L. 1991, A\&A, 242, 256

McClements, K. G. 1987, A\&A, 175, 255

McClements, K. G. 1989, A\&A, 208, 279

Mel'Nik, V. N., Lapshin, V., \& Kontar, E. 1999, Sol. Phys., 184, 353

Melrose, D. B. 1982, Sol. Phys., 79, 173

Pécseli, H. L. 2014, J. Plasma Phys., 80, 745

Ratcliffe, H., \& Kontar, E. P. 2014, A\&A, 562, A57
Rhee, T., Ryu, C.-M., Woo, M., et al. 2009a, ApJ, 694, 618

Rhee, T., Woo, M., \& Ryu, C.-M. 2009b, J. Kor. Phys. Soc., 54, 313

Robinson, P. A., \& Cairns, I. H. 1998, Sol. Phys., 181, 363

Syrovatskii, S. I., \& Shmeleva, O. P. 1972, Sov. Astron., 16, 273

Tang, F., \& Moore, R. L. 1982, Sol. Phys., 77, 263

Tsytovich, V. N. 1967, Sov. Phys. Uspekhi, 9, 805

Umeda, T. 2010, J. Geophys. Res. (Space Phys.), 115, 1204

Wild, J. P. 1950, Austr. J. Sci. Res. A Phys. Sci., 3, 541

Wild, J. P., \& McCready, L. L. 1950, Austr. J. Sci. Res. A Phys. Sci., 3, 387

Yoon, P. H. 2000, Phys. Plasmas, 7, 4858

Yoon, P. H. 2005, Phys. Plasmas, 12, 052313

Yoon, P. H. 2006, Phys. Plasmas, 13, 022302

Zharkova, V. V., \& Siversky, T. V. 2011, ApJ, 733, 33

Zheleznyakov, V. V., \& Zaitsev, V. V. 1970, Sov. Astron., 14, 250

Ziebell, L. F., Gaelzer, R., Pavan, J., \& Yoon, P. H. 2008, Plasma Phys. Controlled Fusion, 50, 085011

Ziebell, L. F., Yoon, P. H., Gaelzer, R., \& Pavan, J. 2014a, ApJ, 795, L32

Ziebell, L. F., Yoon, P. H., Gaelzer, R., \& Pavan, J. 2014b, Phys. Plasmas, 21, 012306

Ziebell, L. F., Yoon, P. H., Simões, F. J. R., Gaelzer, R., \& Pavan, J. 2014c, Phys. Plasmas, 21, 010701

Ziebell, L. F., Yoon, P. H., Petruzzellis, L. T., Gaelzer, R., \& Pavan, J. 2015, ApJ, 806,237 


\section{Appendix A: Fokker-Planck equation coefficients}

Here we specifically follow the notations and conventions that can be found in Gaffey (1976), which can be summarized as follows. Starting from the Landau form of the Fokker-Planck collision operator defined in Eq. (3) and absorbing the ambient density into the definition for particle distribution function, $f_{a}=\hat{n}_{a} F_{a}$, we have

$\theta_{a b}\left(f_{a}, f_{b}\right)=\frac{2 \pi e_{a}^{2} e_{b}^{2} \ln \Lambda}{m_{a}} \frac{\partial}{\partial \boldsymbol{v}_{a}} \cdot \int d^{3} v_{b} \stackrel{\leftrightarrow}{U} \cdot\left(\frac{1}{m_{a}} \frac{\partial}{\partial \boldsymbol{v}_{a}}-\frac{1}{m_{b}} \frac{\partial}{\partial \boldsymbol{v}_{b}}\right) f_{a} f_{a}$,

$\stackrel{\leftrightarrow}{U}=\frac{\partial^{2} w}{\partial \boldsymbol{v}_{a} \partial \boldsymbol{v}_{a}}=\frac{1}{w^{3}}\left(w^{2} \stackrel{\leftrightarrow}{1}-\boldsymbol{w w}\right)$

$w=|\boldsymbol{w}|=\left|\boldsymbol{v}_{a}-\boldsymbol{v}_{b}\right|$.

Integrating by parts we obtain

$$
\begin{aligned}
\theta_{a b}\left(f_{a}, f_{b}\right)= & \frac{2 \pi e_{a}^{2} e_{b}^{2} n_{0 b} \ln \Lambda v_{T b}}{m_{a}^{2}} \frac{\partial}{\partial \boldsymbol{v}_{a}} \\
& \times\left(\frac{\partial f_{a}}{\partial \boldsymbol{v}_{a}} \cdot \frac{\partial^{2} G\left(x_{a b}\right)}{\partial \boldsymbol{v}_{a} \partial \boldsymbol{v}_{a}}-\frac{m_{a}}{m_{b}} f_{a} \frac{\partial}{\partial \boldsymbol{v}_{a}} \cdot \frac{\partial^{2} G\left(x_{a b}\right)}{\partial \boldsymbol{v}_{a} \partial \boldsymbol{v}_{a}}\right),
\end{aligned}
$$

where

$G\left(x_{a b}\right)=\frac{1}{n_{0 b} v_{T b}} \int d^{3} v_{b} f_{b} U_{a b} w_{a b}$,

and $x_{a b} \equiv v_{a} / v_{T b}$, with $v_{T b}$ denoting the thermal velocity for particle species $b, v_{T b}=\left(2 T_{b} / m_{b}\right)^{1 / 2}$. Here, $n_{0 b}$ is the number density of particles of species $b$.

At this point we introduce the simplifying argument that in the beam-plasma instability, the most significant evolution occurs in the tail of the particle distribution function, involving particle densities that are much lower than the background density. If so, then it is justified to assume that the most significant collisional effect will be due to collisions between tail particles with particles of the background distribution. Assuming that the background distribution is a Maxwellian distribution, the function $G$ in Eq. (3) may be written as (Gaffey 1976)

$G\left(x_{a b}\right)=\left(x_{a b}+\frac{1}{2 x_{a b}}\right) \Phi\left(x_{a b}\right)+\frac{1}{2} \Phi^{\prime}\left(x_{a b}\right)$,

where $\Phi\left(x_{a b}\right)$ is the error function, and $\Phi^{\prime}\left(x_{a b}\right)$ its derivative,

$\Phi\left(x_{a b}\right) \equiv \frac{2}{\sqrt{\pi}} \int_{0}^{x} e^{-t^{2}} \mathrm{~d} t$

$\Phi^{\prime}\left(x_{a b}\right)=\frac{2}{\sqrt{\pi}} e^{-x^{2}}$.

Making use of the auxiliary function $\Psi$,

$\Psi(x) \equiv \Phi(x)-x \Phi^{\prime}(x)$,

and performing some simple algebra, one obtains the following form for the collisional term (Gaffey 1976),

$$
\begin{aligned}
\theta_{a b}\left(f_{a}, f_{b}\right)= & \Gamma_{a b}\left[\frac{\partial}{\partial \boldsymbol{v}_{a}} \cdot\left(2 \frac{m_{a}}{m_{b}} \Psi\left(x_{a b}\right) \frac{\boldsymbol{v}_{a}}{v_{a}^{3}} f_{a}\right)\right. \\
& +\frac{\partial}{\partial \boldsymbol{v}_{a}} \cdot\left\{\left[\left(\Phi\left(x_{a b}\right)-\frac{1}{2 x_{a b}^{2}} \Psi\left(x_{a b}\right)\right) \frac{\partial^{2} v_{a}}{\partial \boldsymbol{v}_{a} \partial \boldsymbol{v}_{a}}\right] \cdot \frac{\partial f_{a}}{\partial \boldsymbol{v}_{a}}\right\} \\
& \left.+\frac{\partial}{\partial \boldsymbol{v}_{a}} \cdot\left[\left(\frac{1}{x_{a b}^{2}} \Psi\left(x_{a b}\right) \frac{\boldsymbol{v}_{a} \boldsymbol{v}_{a}}{v_{a}^{3}}\right) \cdot \frac{\partial f_{a}}{\partial \boldsymbol{v}_{a}}\right]\right] .
\end{aligned}
$$

Henceforth, we concentrate on the collision term affecting the electron distribution, so that $f_{a}=f_{\mathrm{e}}$. Let us write the electron velocity simply as $v$, use the non-dimensional variables $\tau=\omega_{\mathrm{pe}} t$ and $\boldsymbol{u}=\boldsymbol{v} / v_{\mathrm{te}}$, and write the term describing binary collisions between electrons and particles of species $b$ as

$$
\begin{aligned}
\theta_{a b}\left(F_{\mathrm{e}}, F_{b}\right) & =(2 \pi) g Z_{b}^{2} \ln \Lambda\left[\frac{\partial}{\partial \boldsymbol{u}} \cdot\left(2 \frac{m_{e}}{m_{b}} \Psi\left(u \frac{v_{t \mathrm{e}}}{v_{t b}}\right) \frac{\boldsymbol{u}}{u^{3}} F_{\mathrm{e}}\right)\right. \\
& +\frac{\partial}{\partial \boldsymbol{u}} \cdot\left\{\left[\left(\Phi\left(u \frac{v_{t \mathrm{e}}}{v_{t b}}\right)-\frac{1}{2 u^{2}} \frac{v_{t b}^{2}}{v_{t \mathrm{e}}^{2}} \Psi\left(u \frac{v_{\mathrm{e}}}{v_{t b}}\right)\right) \frac{\partial^{2} u}{\partial \boldsymbol{u} \partial \boldsymbol{u}}\right] \cdot \frac{\partial F_{\mathrm{e}}}{\partial \boldsymbol{u}}\right\} \\
& \left.+\frac{\partial}{\partial \boldsymbol{u}} \cdot\left[\left(\frac{1}{u^{2}} \frac{v_{t b}^{2}}{v_{t \mathrm{e}}^{2}} \Psi\left(u \frac{v_{t \mathrm{e}}}{v_{t b}}\right) \frac{\boldsymbol{u} \boldsymbol{u}}{u^{3}}\right) \cdot \frac{\partial F_{\mathrm{e}}}{\partial \boldsymbol{u}}\right]\right],
\end{aligned}
$$

where we have considered $n_{\mathrm{e}} \simeq n_{\mathrm{i}}$, introduced $Z_{\mathrm{i}}$ and $Z_{\mathrm{e}}$ as the ion and electron charge number, respectively, with $Z_{\mathrm{i}}=Z_{\mathrm{e}}=1$ and used $g$, as defined in Eq. (6).

In the present application, we consider a 2D system, which serves as a good approximation for the fully 3D case with azimuthal symmetry. In the $2 \mathrm{D}$ case, we make use of $\left(\partial^{2} / \partial u_{x} \partial u_{x}\right) u=u_{z}^{2} / u^{3},\left(\partial^{2} / \partial u_{x} \partial u_{z}\right) u=\left(\partial^{2} / \partial u_{z} \partial u_{x}\right) u=$ $-u_{x} u_{z} / u^{3},\left(\partial^{2} / \partial u_{z} \partial u_{z}\right) u=u_{x}^{2} / u^{3}$ and write Eq. (7) in the following form,

$$
\begin{aligned}
\theta_{a b}\left(F_{\mathrm{e}}, F_{b}\right)= & \frac{\partial}{\partial u_{x}}\left(a_{x}^{b} F_{\mathrm{e}}+b_{x x}^{b} \frac{\partial F_{\mathrm{e}}}{\partial u_{x}}+b_{x z}^{b} \frac{\partial F_{\mathrm{e}}}{\partial u_{z}}\right) \\
& +\frac{\partial}{\partial u_{z}}\left(a_{z}^{b} F_{\mathrm{e}}+b_{z x}^{b} \frac{\partial F_{\mathrm{e}}}{\partial u_{x}}+b_{z z}^{b} \frac{\partial F_{\mathrm{e}}}{\partial u_{z}}\right),
\end{aligned}
$$

where

$$
\begin{aligned}
& a_{i}^{b}=(2 \pi) g Z_{b}^{2} \ln \Lambda\left[2 \frac{m_{\mathrm{e}}}{m_{b}} \Psi\left(u \frac{v_{t \mathrm{e}}}{v_{t b}}\right) \frac{u_{i}}{u^{3}}\right], \\
& \left(\begin{array}{l}
b_{x x}^{b} b_{x z}^{b} \\
b_{z x}^{b} b_{z z}^{b}
\end{array}\right)=\frac{\Gamma_{e j}}{\omega_{\mathrm{pe}} v_{t \mathrm{e}}^{3}} \frac{1}{u^{5}}\left\{\left[u^{2} \Phi\left(u \frac{v_{t \mathrm{e}}}{v_{t b}}\right)-\frac{1}{2} \frac{v_{t b}^{2}}{v_{t \mathrm{e}}^{2}} \Psi\left(u \frac{v_{t \mathrm{e}}}{v_{t b}}\right)\right]\right. \\
& \left.\times\left(\begin{array}{c}
u_{z} u_{z}-u_{x} u_{z} \\
-u_{x} u_{z} u_{x} u_{x}
\end{array}\right)+\frac{v_{t b}^{2}}{v_{t \mathrm{e}}^{2}} \Psi\left(u \frac{v_{t \mathrm{e}}}{v_{t b}}\right)\left(\begin{array}{cc}
u_{x} u_{x} & -u_{x} u_{z} \\
-u_{x} u_{z} & u_{z} u_{z}
\end{array}\right)\right\} .
\end{aligned}
$$

In addition, it is easy to verify that the equation for the electron distribution function, obtained from (6), can be written for the case $2 \mathrm{D}$ in the following form:

$$
\begin{aligned}
\frac{\partial F_{\mathrm{e}}}{\partial \tau}= & \frac{\partial}{\partial u_{x}}\left(A_{x}^{\mathrm{el}} F_{\mathrm{e}}+D_{x x}^{\mathrm{el}} \frac{\partial F_{\mathrm{e}}}{\partial u_{x}}+D_{x z}^{\mathrm{el}} \frac{\partial F_{\mathrm{e}}}{\partial u_{z}}\right) \\
& +\frac{\partial}{\partial u_{z}}\left(A_{z}^{\mathrm{el}} F_{\mathrm{e}}+D_{z x}^{\mathrm{el}} \frac{\partial F_{\mathrm{e}}}{\partial u_{x}}+D_{z z}^{\mathrm{el}} \frac{\partial F_{\mathrm{e}}}{\partial u_{z}}\right) \\
& +\sum_{b} \theta_{a b}\left(F_{\mathrm{e}}, F_{b}\right),
\end{aligned}
$$

where, as a further and usual approximation, we have neglected the effect of $S$ waves. Making use of $\mu_{\boldsymbol{q}}^{L}=1$, the coefficients $A_{i}^{\mathrm{e}}$ and the $D_{i j}^{\mathrm{e}}$ can be written as

$$
\begin{aligned}
& A_{i}^{\mathrm{el}}=g \int_{-\infty}^{\infty} \mathrm{d} q_{x} \int_{-\infty}^{\infty} \mathrm{d} q_{z} \frac{q_{i}}{q_{x}^{2}+q_{z}^{2}} \sum_{\sigma= \pm 1} \sigma z_{\boldsymbol{q}}^{L} \delta\left(\sigma z_{\boldsymbol{q}}^{L}-q_{x} u_{x}-q_{z} u_{z}\right), \\
& D_{i j}^{\mathrm{el}}=\int_{-\infty}^{\infty} \mathrm{d} q_{x} \int_{-\infty}^{\infty} \mathrm{d} q_{z} \frac{q_{i} q_{j}}{q_{x}^{2}+q_{z}^{2}} \sum_{\sigma= \pm 1} \mathcal{E}_{\boldsymbol{q}}^{\sigma L} \delta\left(\sigma z_{\boldsymbol{q}}^{L}-q_{x} u_{x}-q_{z} u_{z}\right) .(\mathrm{A} .12)
\end{aligned}
$$

